Br. J. clin. Pharmac. (1988), 26, 7-11

\title{
Spontaneous reporting of adverse drug reactions II: Uses
}

\author{
M. D. RAWLINS \\ Wolfson Unit of Clinical Pharmacology, University of Newcastle upon Tyne, Newcastle upon Tyne, NE2 3LH
}

Despite its inherent limitations, discussed previously (Rawlins, 1988), the UK yellow card scheme has made substantial contributions to drug safety. Although this review concentrates on the British experience, it must be stressed that other national schemes have made equally important contributions especially those in Scandinavia, Holland, Australia and New Zealand, and North America. The schemes in West Germany, Spain and France, as a result of recent changes, are likely to become more prominent in the future.

\section{Identification of iatrogenic hazards}

Isolated reports of adverse drug reactions have occasionally been so obvious and compelling that commercial or regulatory action has followed almost immediately. Thus, a single report of mercury poisoning led to the abandonment of a proprietary preparation as treatment for nappy rash (Inman, 1980). More recently, the reported inhalation of part of the delivery device of a metered aerosol inhaler resulted in its withdrawal, by the manufacturer, within $48 \mathrm{~h}$.

Such instances, however, are the exception rather than the rule and the recognition of novel iatrogenic hazards, from yellow card reports, depends largely on the weight of available evidence. The main features used in recognising novel adverse reactions include the nature of the reaction; the pharmaceutical, pharmacological and toxicological properties of the compound; and the numbers of reports, both in relation to the frequency with which the adverse event might be expected amongst recipients of the drug, and to its usage. A few clinical conditions or syndromes (including acute anaphylaxis and acute dystonias and dyskinesias) are almost invariably iatrogenic. Others are so unusual in a particular age group (parkinsonism in young adults) or gender (gynaecomastia in otherwise healthy males) as to suggest strongly the possibility of an iatrogenic basis where they occur in association with the use of a particular drug. Relatively uncommon disorders such as aplastic and haemolytic anaemia, neutropenia, erythema multiforme, Guillain-Barre syndrome or fibrosing alveolitis may be recognised as iatrogenic hazards where there are clusters of reports amongst patients receiving the same product.

Adverse events which are manifestations of the known pharmacological or toxicological action of a particular drug, and which are thus potential Type A adverse reactions (Rawlins \& Thompson, 1985), may be recognised as such if the temporal relationships are appropriate, and the event is uncommon in the treated population. Examples include hypotension with prazosin or angiotensin converting-enzyme inhibitors, hypoglycaemia with antidiabetic agents, or bleeding with anticoagulants or thrombolytic agents. Problems of interpretation may arise where the adverse event, though possibly a Type A reaction, is also frequent in the treated population. Spontaneous reports of arrhythmias associated with anti-arrhythmic agents, or of unstable angina with calcium-channel blockers, are thus difficult to evaluate even when they are frequent.

The UK spontaneous reporting scheme has successfully provided 'early warnings' of a number of drug hazards and examples are shown in Table 1. Although such warnings may have been preceded, in some instances, by publication of single case-reports of suspicious associations, the yellow card data provided a sufficient weight of evidence to indicate particular hazards. On occasions, a small number of reports of suspected adverse reactions have prompted detailed epidemiological or clinical studies to confirm or refute the possibility of a causal association (Inman, 1980). Early warnings based on UK yellow card data have, in some cases, been paralleled by similar signals from other national schemes in Europe or North America and vice versa.

\section{Characterisation of iatrogenic syndromes}

A yellow card report provides information about the patient (age, sex and indication for treatment), the drug (dose and duration of treatment), and the reaction (its clinical and pathological features, reaction interval and outcome). Careful study of a series of reports of single (or closely related) reactions may serve to identify diagnostic features or suggest pathogenetic 
Table 1 Early warnings of suspected adverse reactions from the CSM's adverse reaction register

\begin{tabular}{lll}
\hline Date & \multicolumn{1}{c}{ Product } & \multicolumn{1}{c}{ Reaction } \\
\hline 1965 & Nifenazone & Blood dyscrasias \\
1965 & Mefenamic acid & Diarrhoea \\
1965 & Ibufenac & Hepatotoxicity \\
1973 & Erythromycin estolate & Hepatotoxicity \\
$1975 \mathrm{a}$ & Prazosin & Hypotension \\
$1975 \mathrm{~b}$ & Hormonal pregnancy tests & Congenital abnormalities \\
$1975 \mathrm{c}$ & Tetracosactrin & Anaphylaxis \\
$1975 \mathrm{~d}$ & Metoclopramide & Extrapyramidal reactions (1) \\
1977 & Topical neomycin & Deafness \\
$1978 \mathrm{a}$ & Nitrofurantoin & Hepatoxicity \\
$1978 \mathrm{~b}$ & Emepromium bromide & Oesophageal ulceration \\
1979 & Diflunisal & Stevens-Johnson syndrome \\
$1981 \mathrm{a}$ & Mebhydrolin & Granulocytopenia (2) \\
$1981 \mathrm{~b}$ & Mianserin & Blood dyscrasias \\
1982 & Amiodarone & Fibrosing alveolitis hepatotoxicity \\
$1983 \mathrm{~b}$ & Zimeldine & Guillain-Barre syndrome (3) \\
$1983 \mathrm{c}$ & Osmosin & Gastrointestinal perforation \\
1985 & Actifed & Hallucinations \\
$1986 \mathrm{a}$ & Nifedipine & Gum hyperplasia (4) \\
$1987 \mathrm{a}$ & Pivmecillinam & Oesophageal ulcer (3) \\
$1987 \mathrm{~b}$ & Propofol & Convulsions \\
\hline
\end{tabular}

Notes (1) Also reported in Argentina

(2) Also reported in Australia and Japan

(3) Also reported in Sweden

(4) Also reported in Israel and Holland

mechanisms. More importantly, however, such studies may indicate measures that would reduce the incidence or severity of the reaction. Analyses of yellow card reports which are intended to characterise specific reactions may, however, be confounded if they fail to take into account the pattern of use. Thus a preponderance of reports of reactions occurring at higher doses, or in particular age groups, do not provide sufficient evidence of dose- or age-related phenomena unless shown to be disproportionate to their prescription and volume.

Examples where spontaneous adverse reaction reports have provided valuable material for the clinical delineation of specific syndromes include nitrofurantoin induced eosinophilic pulmonary reactions (Penn \& Griffin, 1982) and the hepatotoxicity associated with ketoconazole (LakeBakaar et al., 1987). Studies of the interval between successive exposures to halothane, and of the increased mortality from hepatotoxicity with the number of exposures, has led to important changes in its use (Inman \& Muskin, 1978; Committee on the Safety of Medicines, 1986d). A recent analysis of reports of anaphylaxis and bronchospasm following the administration of desensitising vaccines (Committee on the Safety of Medicines Update, 1986c; Rawlins et al., 1988) resulted in the Committee advising doctors of special precautions that should be taken by doctors undertaking in this form of treatment.

Perhaps the most important contributions made by the UK yellow card scheme have been those concerned with establishing the effects of dose, age and gender on drug toxicity. The statistical basis for investigating the effect of single factors was originally described by Finney (1971). In collaboration with the Swedish and Danish authorities, Inman and his colleagues (1970) showed that there was an excess of reports of thromboembolic phenomena amongst women using higher doses of oestrogens than expected from sales figures. Products containing high doses of oestrogens were consequently withdrawn from the market, both in the UK, and elsewhere. Data derived from spontaneous reports of adverse reactions, leading to dosage changes, include those involving prazosin (Committee on the Safety of Medicines, 1975a) and enalapril (Committee on the Safety of Medicines, 1986b).

Age and gender are important correlates with some, though not all (Woodhouse et al., 1986), adverse reactions. By analysing adverse reaction reporting rates, and prescribing figures at various ages, it has been possible to demonstrate the susceptibility of the elderly to blood dyscrasias 
with both mianserin (Committee on the Safety of Medicine, 1983a) and co-trimoxazole (Committee on the Safety of Medicines, 1985b). More recently Simpson et al. (1987) have developed Finney's (1971) methods to test the effects of two or more factors simultaneously by using generalised linear models. With this technique an analysis of reports of extrapyramidal reactions with metoclopramide (Bateman et al., 1985) indicated that acute reactions (dystonias, dyskinesias) were more frequent in adolescents and young adults whilst chronic reactions (parkinsonism) were more likely in the elderly. The data also suggested that the risk of acute dystoniasdyskinesias was greater in young females than males. A study of extrapyramidal reactions to prochlorperazone and metoclopramide yielded comparable results (Bateman et al., 1986) except that no discernable effect of gender was detectable.

\section{Comparative toxicity within therapeutic groups}

Use of the yellow card system to assess the relative toxicities of products with the same therapeutic class has evoked scientific controversy (Sacks \& Bortnichak, 1986) because of the obvious potential for confounding and bias that was discussed in the previous article (Rawlins, 1988). For this reason the CSM has been cautious in using such evidence when advising severe regulatory action against individual products unless the evidence has been compelling, and cannot be reasonably explained by factors other than those of enhanced toxicity.

The earliest attempts at comparisons of drug toxicity within therapeutic groups, reviewed recently by Speirs (1986), were by means of 'profiling'. In this technique the 'profile' of the proportion of the total number of reports to a particular drug, attributable to each of the major organ-system, is compared to other products within the same class. Similar 'profiles' can be constructed to individual reactions within a single organ-system. Profiles demonstrated the hepatotoxicity of ibufenac (withdrawn in 1967) and the myelotoxicity of the butazones (Cuthbert, 1977). The technique also suggested the liability of one particular tricyclic antidepressant (protriptyline) to produce photosensitivity (Inman, 1980). Whilst 'profiling' can be a useful approach to comparing the toxicity of two, or more, products it has important limitations. First, it assumes that toxicity within a therapeutic class is distributed homogeneously between individual products: this cannot, however, be necessarily assumed, and for some compounds an apparent increase in the proportion of reactions involving one particular organ-system may merely reflect an absence of reactions in others. Second, 'profiling' does not identify an overall (homogeneous) increase in the tc ricity of one product compared with others.

An alternative to 'profiling', which has been used by regulatory authorities in Scandinavia, North America and New Zealand, as well as by the CSM, is to express reporting rates in relation to prescription (or sales) volume, for comparable marketing periods. Using this methodology the toxicities of zimeldine (hepatotoxicity, peripheral neuropathy, Guillain-Barre syndrome) and of nomifensine (hepatotoxicity, haemolytic anaemia), in relation to their usage, were shown to be substantially greater than other marketed antidepressants and both drugs have been withdrawn from the market (Committee on the Safety of Medicines, 1986f). Similar analyses of reports, to the Swedish regulatory authority, of lactic acidosis with biguanide antidiabetic agents (Bergman et al., 1978) lead to the withdrawal of phenformin in Scandinavia and elsewhere.

In recent years, one quarter of all yellow card reports have incriminated NSAIDs despite the fact that these drugs account for only $5 \%$ of NHS prescriptions. Careful studies of reporting rates, corrected for prescription volume and marketing life, indicated to the CSM that they fell broadly into three categories (Committee on the Safety of Medicines, 1986e): five products (benoxaprofen, indoprofen, fenclofenac, feprazone and Osmosin) showed increased reporting rates and have been withdrawn; one product (ibuprofen) appeared safer-at least at low dosage - and has been licensed for 'pharmacy' sale; the toxicity of the others could not be distinguished, and remain available as 'prescription only medicines'. It is important to appreciate, however, that this analysis inevitably excluded NSAIDs introduced before the yellow card scheme started (aspirin, indomethacin, mefenamic acid and flufenamic acid) since the influence of product life on reporting rates cannot be assessed.

\section{Continued monitoring of drug safety}

One of the special features of the UK spontaneous reporting system is its potential for monitoring the safety of individual drugs throughout their marketing lives. Thus, continued reports of blood dyscrasias with oxyphenbutazone and phenylbutazone, and of lactic acidosis with phenformin, provided evidence of the continuing toxicity of these products. By contrast, the increasing 
number of reports of haemolytic anaemia with nomifensine (Committee on the Safety Medicine, 1986f), occurring over a period of 6 years after marketing, alerted both the Committee and the manufacturer to the drugs's latent dangers. Changes in the reporting of adverse reactions may also provide a useful basis for monitoring the effects of regulatory action and advice that stops short of withdrawal (e.g. restricted indications, altered dosage regimens).

\section{The future}

The CSM's yellow card system plays a central role in monitoring the safety of marketed pharmaceutical products and is complimentary to other methods for postmarketing surveillance (Rawlins, 1987). It has successfully identified, and characterised, many acute drug hazards and it can (with care) provide estimates of relative risk even though its value in determining absolute risk is small. For the future, however, further development of the scheme is necessary if its fullest potential is to be achieved. First, and most importantly, reporting rates need to increase; higher reporting rates might reduce the chances of bias, would certainly provide earlier indications of iatrogenic hazards and would improve overall surveillance. This especially applies to the reporting of severe reactions. Since these are often seen in hospital, attempts to improve reporting by hospital medical staff has a high priority; clinical pharmacologists and hospital pharmacists could do much to help. Second, the CSM's Adverse Reaction Register is a national epidemiological asset that should be readily available for reference, and as source material for bona fide research by investigators unconnected with the CSM. The Committee is therefore making the Register more easily available to external enquiries by means of Viewdata, and is planning a new computer system for better access to its data-base. Finally, the Committee is experimenting with modifications to its reporting system. The Regional Monitoring Centres in the Wales, and in the West Midlands and the Northern Regions, have been established for some years, and a 'prompted' reporting system (the Red Alert Scheme) for patients prescribed new chemical entities, is being evaluated in collaboration with the Drug Safety Research Unit. The Committee is also investigating special reporting schemes with dermatologists (through the Institute of Dematology) and with anaesthetists (through the Association of Anaesthetists).

In 1989 the Committee celebrates the silver jubilee of its yellow card scheme. Clinical pharmacologists have a professional responsibility to help ensure that the scheme's contributions to drug safety are even greater during the next 25 years.

\section{References}

Bateman, D. N., Rawlins, M. D. \& Simpson, J. M. (1985). Extrapyramidal reactions with metoclopramide . Br. med. J., 291, 930-932.

Bateman, D. N., Rawlins, M. D. \& Simpson, J. M. (1986). Extrapyramidal reactions to prochlorperazine and haloperidol in the United Kingdom. Quart. J. Med., 59, 549-556.

Bergman, U., Boman, G. \& Wilholm, B-E. (1978). Epidemiology of adverse drug reactions to phenformin and metoformin. Br. med. J., 2, 464-466.

Committee on the Safety of Drugs (1965). Some antiarthritic drugs and a new analgesic. Adverse Reactions Series No 3.

Committee on the Safety of Medicines (1973). Jaundice $\&$ erythromycin estolate. Adverse Reactions Series No. 10.

Committee on the Safety of Medicines (1975a). Prazosin and loss of consciousness. Adverse Reactions Series No. 12.

Committee on the Safety of Medicines (1975b). Hormonal pregnancy tests: association with congenital abnormalities. Adverse Reactions Series No. 13.

Committee on the Safety of Medicines (1975c). Reactions with tetracosactin. Current Problems No. 1.

Committee on the Safety of Medicines (1975d). Extrapyramidal reaction to metoclopramide. Current Problems No. 1.

Committee on the Safety of Medicines (1977). Neomycin and deafness. Current Problems No. 2.

Committee on the Safety of Medicines (1978a). Nitrofurantoin and hepatoxicity. Current Problems No. 3.

Committee on the Safety of Medicines (1978b). Emepromium bromide and oesophageal ulceration. Current Problems No. 3.

Committee on the Safety of Medicines (1979). Diflunisal (Dolobid) and Stevens-Johnson syndrome. Current Problems No. 4.

Committee on the Safety of Medicines (1981a). Mebhydolin (Fabahistine) and white cell depression. Current Problems No. 7.

Committee on the Safety of Medicines (1981b). Mianserin (Bolvidon, Norval) and blood dyscrasias. Current Problems No. 7.

Committee on the Safety of Medicines (1982). Amiodarone (Cordarone X). Current Problems No. 8.

Committee on the Safety of Medicines (1983a). Mianserin (Bolvidon, Norval). Current Problems No. 10.

Committee on the Safety of Medicines (1983b). Zimel- 
dine (Zelmid). Current Problems No. 11.

Committee on the Safety of Medicines (1983b). Osmosin (controlled release indomethacin). Current Problems No. 11.

Committee on the Safety of Medicines (1985a). Actifed syrup and hallucinations in children. Current Problems No. 14.

Committee on the Safety of Medicines (1985b). Deaths associated with cotrimoxazole, ampicillin and trimethoprim. Current Problems No. 15.

Committee on the Safety of Medicines (1986a). Nifedipine and gum hyperplasia. Current Problems No. 17.

Committee on the Safety of Medicines (1986b). Adverse reactions to enalapril (Innovace). Current Problems No. 17.

Committee on the Safety of Medicines (1986c). CSM Update: Desensitising vaccines. Br. med. J., 293, 948.

Committee on the Safety of Medicines (1986d). Halothane hepatotoxicity. Current Problems No. 18.

Committee on the Safety of Medicines (1986e). CSM Update: Non-steroidal anti-inflammatory drugs and serious gastrointestinal adverse reactions 2 . Br. med. J., 292, 1190-1191.

Committee on the Safety of Medicines (1986f). CSM Update: Withdrawal of nomifensin. Br. med. J., 293, 41.

Committee on the Safety of Medicines (1987a). Pivmecillinam and oesophageal injury. Current Problems No. 19.

Committee on the Safety of Medicines (1987b). Propofol. Current Problems No. 20.

Cuthbert, M. F. (1977). Adverse reactions to antirheumatic drugs: some correlations with animal toxicity studies. In Current approaches in toxicology ed. Ballantyne, B. Wright: Bristol.

Finney, D. J. (1971). Statistical logic in the monitoring of adverse reactions to therapeutic drugs. Methods of Information in Medicine, 10, 237-242.

Inman, W. H. W. (1980). The United Kingdom. In Monitoring for Drug Safety ed. Inman, W. H. W., pp. 9-47. 1st edition. Lancaster: MTP Press.

Inman, W. H. W. \& Muskin, W. W. (1978). Jaundice after halothane: a further report. Br. med. J., 2, 1455-1457.

Inman, W. H. W., Rawson, N. S. B. \& Witton, L. V. (1986). Prescription-event monitoring. In Monitor- ing for drug safety ed. Inman, W. H. W., pp. 213235. 2nd edition. Lancaster: MTP Press.

Inman, W. H. W., Vessey, M. P., Westerholm, B. \& Engelund, A. (1970). Thromboembolic disease and the steroidal content of oral contraceptives. Br. med. J., 2, 203-205.

Inman, W. H. W. (1986). The United Kingdom. In Monitoring for drug safety, ed. Inman, W. H. W., pp. 13-48. 2nd edition. Lancaster: MTP Press.

Lake-Bakaar, G., Scheuer, P. J. \& Sherlock, S. (1987) Hepatic reactions associated with ketoconazole in the United Kingdom. Br. med. J., 294, 419-422.

Penn, R. G. \& Griffin, J. P. (1982). Adverse reactions to nitrofurantoin in the United Kingdom, Sweden and Holland. Br. med. J., 284, 1440-1442.

Rawlins, M. D. (1987). Advantages and disadvantages of different techniques of post-marketing surveillance. In Adverse drug reactions ed. Mann, R. D., pp. 67-74. Carnforth: Parthenon.

Rawlins, M. D. (1988). Spontaneous reporting of adverse drug reactions. 1 . The data. Br. J. clin. Pharmac., 26, 1-5.

Rawlins, M. D. \& Thompson, J. W. (1985). Mechanisms of adverse drug reactions. In Textbook of adverse drug reactions, ed. Davies, D. M. pp. 1238. Oxford: Oxford University Press.

Rawlins, M. D., Wood, S. M. \& Mann, R. D. (1988). Hazards with desensitising agents. In Regulatory control of standardisation of allergenic extracts, (in press).

Sachs, R. M. \& Bortnichak, E. A. (1986). An evaluation of spontaneous adverse drug reaction monitoring systems. Am. J. Med., 81 (Suppl 5B), 59-65.

Simpson, J. M., Bateman, D. N. \& Rawlins, M. D. (1987). Using the adverse reactions register to study the effects of age and sex on adverse drug reactions. Statistics in Medicine, 6, 863-867.

Speirs, C. J. (1986). Prescription-related adverse reaction profiles and their use in risk-benefit analysis. In Iatrogenic disease eds D'Arcy, P. D. \& Griffin, J. P., pp. 93-101. Oxford: Oxford University Press.

Woodhouse, K. W., Mortimer, O. \& Wilholm, B-E. (1986). Hepatic adverse reactions: the effects of age. In Liver and Aging 1986 ed. Kitani, K., pp. 7580. Elsevier: Amsterdam.

(Received 11 January 1988, accepted 23 March 1988) 